\title{
Impact of increasing threshold level on higher bit rate in free space optical communications
}

\begin{abstract}
The biggest challenge facing free space optical deployment is optical signal propagation in different atmospheric conditions such as fog, low clouds, rain, snow, dust, haze and various combinations of each. A transmission and detection technique for free space optical communications is proposed where it employs two beams, one modulated with the data, the other with the inverted version of the same data. A differential detection technique is used at the receiver whereby the inverted data are used as the reference to perform the decision making, as opposed to the fixed threshold used in the conventional technique. The probability of error under threshold instability in the proposed differential technique and Intensity Modulation/Direct Detection (IM/DD) technique are compared. A simulation under heavy rainfall condition of $8.33 \times 10 \overline{1} 4 \mathrm{~cm} / \mathrm{sec}$ at bit rate of $155 \mathrm{Mbps}$ to $10 \mathrm{Gbps}$ with $0 \mathrm{dBm}$ of launch power and $1.5 \mathrm{~km}$ distance shows that this technique achieves an improvement compared to the conventional IM/DD. The differential detection can support a higher bit rate (up to $9 \mathrm{Gbps}$ ) because of its noise reduction capabilities due to higher threshold level implemented in the receiver. The IM/DD in contrast, can support bit rates of only 2.5 Gbps. This analysis focuses on weather conditions in Malaysia.
\end{abstract}

Keyword: Differential detection; Intensity modulation direct detection; Free space optical; Non-selective scattering; Probability of error 